\title{
ON DOUBLE SHEHU TRANSFORM AND ITS PROPERTIES WITH APPLICATIONS
}

\section{SULIMAN ALFAQEIH*, EMINE MISIRLI}

\author{
Ege University, Faculty of Science, Department of Mathematics, 35100, Turkey
}

*Corresponding author: alfaqeihsuliman@gmail.com

\begin{abstract}
In the current paper, we have generalized the concept of one dimensional Shehu transform into two dimensional Shehu transform namely, double Shehu transform (DHT). Further, we have established some main properties and theorems related to the (DHT). To show the efficiency, high accuracy and applicability of the proposed transform, we have implemented the new transform to solve integral equations and partial differential equations.
\end{abstract}

\section{INTRODUCTION}

Many problems in the fields of most applied science and engineering encounter double integral equations or partial differential equations describing the physical phenomena [20-22]. Solving such equations using single transforms is more difficult than using the double transforms. In the past few decades, integral transforms have been the focus of many authors due to their huge appearance in various applications in modern sciences and engineering, in the current years, a very extensive literature on integral transforms of a function of one but there is a little work available on double integral transform. Consequently, great attention has been given to deal with the double integral transform.

Among the solution methods, integral transform methods are rather and popular, Hence in the literature, there are many different types of integral transforms such as Fourier transform [1] Laplace $[2,3]$ transform, Sumudu transform [4-6,19], Natural transform [7], Ezaki transform [8], and so on. These kinds of transforms

Received February $16^{\text {th }}, 2020$; accepted March $13^{\text {th }}, 2020$; published May $1^{\text {st }}, 2020$.

2010 Mathematics Subject Classification. 44A10, 44A20, 44A30, 44A35.

Key words and phrases. partial differential equation (PDE); telegraph equation; integral equations; Sumudu transform (ST); Laplace transforms (LT); Shehu transform (HT).

(C)2020 Authors retain the copyrights of their papers, and all open access articles are distributed under the terms of the Creative Commons Attribution License. 
have a wide variety of applications in various areas in applied physics, applied mathematics, statistics, engineering and in most of other sciences $[9,10]$.

Shehu transform (HT) of single variable [11], is a new transform which was recently introduced by Shehu Maitama and Weidong Zhao in 2019. (HT) is a generalization of Laplace and Sumudu transforms. This transform is used to solve both ordinary and partial differential equations. After the appearance of the (HT), many authors applied this transform to solve partial differential equations including ordinary and fractional, for instance, see $[12-15,20]$.

The main objective of this paper is to extend the one dimensional Shehu integral transform to two dimensional Shehu integral transform namely double Shehu transform and to get the solution of initial and boundary value problems in different areas of real life science and engineering.

This work is organized as follows. In section 2, we present some notations about Laplace, sumudu, and single Shehu transforms. We then introduce the definition of double Shehu transform and its inverse with examples, and we prove the existence and uniqueness theorems of the new transform in section 3 . In section 4 , we discuss some properties and theorems related to the double Shehu transform. in section 5 , we implement the double Shehu transform method to some examples of double integral equations with convolution and partial differential equations. Section 6 is for the conclusions of this paper.

\section{Preliminaries}

In this section, we present some basic notations about the Laplace, Sumudu and the Shehu transforms.

Definition 2.1.Let $g:(0, \infty) \rightarrow \Re$ be a real valued function. The single Laplace transform of $g$ is defined by:

$$
G(p)=L(g(x): p)=\int_{0}^{\infty} e^{-p x} g(x) d x, p \in \mathrm{C}
$$

Definition 2.2. [16] Let $g(x, t), \mathrm{x}, \mathrm{t}>0$ be a real valued function. The double Laplace transform of $g$ is defined by:

$$
L_{x} L_{t}(g(x, t):(p, q))=G(p, q)=\int_{0}^{\infty} \int_{0}^{\infty} e^{-(p x+q t)} g(x, t) d x d t, \mathrm{p}, \mathrm{q} \in \mathrm{C}
$$

Definition 2.3. Let $g:(0, \infty) \rightarrow \Re$ be a real valued function. The single Sumudu transform of $g$ is defined by:

$$
G(p)=S(g(x): p)=\int_{0}^{\infty} e^{-x} g(p x) d x, p \in \mathrm{C}
$$

Definition 2.4. [17] Let $g(x, t)$ be a real valued function. The double Sumudu transform of $g$ is defined by: 


$$
S_{x} S_{t}(g(x, \tau):(p, q))=G(p, q)=\int_{0}^{\infty} \int_{0}^{\infty} e^{-x-t} g(p x, q t) d x d t .
$$

Definition 2.5. [11] The single Shehu transforms (ST) of a real valued function $g(x, t)$ with respect to the variables $x$ and t respectively, are defined by:

$$
\begin{aligned}
& H_{x}(g(x, t): p, u)=\int_{0}^{\infty} e^{-\frac{p x}{u}} g(x, t) d x, \\
& H_{t}(g(x, t): q, v)=\int_{0}^{\infty} e^{-\frac{q t}{v}} g(x, t) d t .
\end{aligned}
$$

Definition 2.6. [11] The inverse Shehu transform $g(x, t)=H_{x t}^{-2}\left[H_{x t}^{2}(g(x, t))\right]$ is defined by the complex integral formula

$$
g(x, t)=H_{x t}^{-2}\left[H_{x t}^{2}(g(x, t))\right]=\frac{1}{2 \pi i} \int_{\alpha-i \infty}^{\alpha+i \infty} \frac{1}{u} e^{\left(\frac{p x}{u}\right)} d p \frac{1}{2 \pi i} \int_{\gamma-i \infty}^{\gamma-i \infty} \frac{1}{v} e^{\left(\frac{q t}{v}\right)} H_{x t}(g(x, t)) d q .
$$

\section{Double Shehu transforms (DHT)}

In this section, we introduce the definitions of (DHT) and the inverse of (DHT).

Definition 3.1. The (DHT) of the function $g(x, t)$ is defined by the double integral as :

$$
H_{x t}^{2}(g(x, t))=G[(p, q),(u, v)]=\int_{0}^{\infty} \int_{0}^{\infty} e^{-\left(\frac{p x}{u}+\frac{q t}{v}\right)} g(x, t) d x d t
$$

on the set of functions

$$
\Omega=\left\{g(x, t): \exists K, \lambda_{1}, \lambda_{2}>0,|g(x, t)|<K \exp \left(\frac{|x+t|}{\lambda_{j}^{2}}\right), j=1,2 \text { and }(x, t) \in R_{+}^{2}\right\},
$$

provided that the integral exist.

Definition 3.2. The inverse double Shehu transform $g(x, t)=H_{x t}^{-2}\left[H_{x t}^{2}(g(x, t))\right]$ is defined by the complex double integral formula

$$
g(x, t)=H_{x t}^{-2}\left[H_{x t}^{2}(g(x, t))\right]=\frac{1}{2 \pi i} \int_{\alpha-i \infty}^{\alpha+i \infty} \frac{1}{u} e^{\left(\frac{p x}{u}\right)} d p \frac{1}{2 \pi i} \int_{\gamma-i \infty}^{\gamma-i \infty} \frac{1}{v} e^{\left(\frac{q t}{v}\right)} H_{x t}^{2}(g(x, t)) d q .
$$

\subsection{Existence and uniqueness of (DHT).}

Theorem 3.1. Let $g(x, t)$ be a continuous function on every finite intervals $(0, X)$ and $(0, T)$, and of exponential order, that is for some $a, b \in \Re$

$$
\sup _{x, t>0} \frac{|g(x, t)|}{e^{(a x+b t)}}<\infty
$$

Then the $(D H T)$ of $g(x, t)$ exists. 
Proof. Using definition of DHT, we have

$$
\begin{aligned}
\left|H_{x t}^{2}(g(x, t))\right| & =\left|\int_{0}^{\infty} \int_{0}^{\infty} e^{-\left(\frac{p x}{u}+\frac{q t}{v}\right)} g(x, t) d x d t\right| \\
& \leq \int_{0}^{\infty} \int_{0}^{\infty} e^{-\left(\frac{p x}{u}+\frac{q t}{v}\right)}|g(x, t)| d x d t \\
& \leq K \int_{0}^{\infty} \int_{0}^{\infty} e^{-\left(\frac{p x}{u}-a x\right)-\left(\frac{q t}{v}-b t\right)} d x d t \\
& =K \int_{0}^{\infty} e^{-\left(\frac{p}{u}-a\right) x} d x \int_{0}^{\infty} e^{-\left(\frac{q}{v}-b\right) t} d t \\
& =\frac{K u v}{(p-a u)(q-b v)} .
\end{aligned}
$$

Theorem 3.2. Let $h(x, t)$ and $l(x, t)$ be continuous functions and having the double Shehu transforms $H_{x t}^{2}(h(x, t))$ and $H_{x t}^{2}(l(x, t))$ respectively. If $H_{x t}^{2}(h(x, t))=H_{x t}^{2}(l(x, t))$ then $h(x, t)=l(x, t)$.

Proof. Assume $\alpha$, and $\gamma$ to be sufficiently large, then since

$$
g(x, t)=H_{x t}^{-2}\left[H_{x t}^{2}(g(x, t))\right]=\frac{1}{2 \pi i} \int_{\alpha-i \infty}^{\alpha+i \infty} \frac{1}{u} e^{\left(\frac{p x}{u}\right)} d p \frac{1}{2 \pi i} \int_{\gamma-i \infty}^{\gamma-i \infty} \frac{1}{v} e^{\left(\frac{q t}{v}\right)} H_{x t}^{2}(g(x, t)) d q,
$$

we deduce that

$$
\begin{aligned}
h(x, t) & =\frac{1}{2 \pi i} \int_{\alpha-i \infty}^{\alpha+i \infty} \frac{1}{u} e^{\left(\frac{p x}{u}\right)} d p \frac{1}{2 \pi i} \int_{\gamma-i \infty}^{\gamma-i \infty} \frac{1}{v} e^{\left(\frac{q t}{v}\right)} H_{x t}^{2}(h(x, t)) d q \\
& =\frac{1}{2 \pi i} \int_{\alpha-i \infty}^{\alpha+i \infty} \frac{1}{u} e^{\left(\frac{p x}{u}\right)} d p \frac{1}{2 \pi i} \int_{\gamma-i \infty}^{\gamma-i \infty} \frac{1}{v} e^{\left(\frac{q t}{v}\right)} H_{x t}^{2}(l(x, t)) d q \\
& =l(x, t),
\end{aligned}
$$

and the theorem is established.

\subsection{Double Shehu transform of some functions:}

(1) If $g(x, t)=1$, then

$$
H_{x t}^{2}(g(x, t))=\int_{0}^{\infty} \int_{0}^{\infty} e^{-\left(\frac{p x}{u}+\frac{q t}{v}\right)} d x d t=\frac{u v}{p q} .
$$

(2) If $g(x, t)=e^{(a x+b t)}$, then

$$
\begin{aligned}
H_{x t}^{2}(g(x, t)) & =\int_{0}^{\infty} \int_{0}^{\infty} e^{-\left(\frac{p x}{u}+\frac{q t}{v}\right)} e^{(a x+b t)} d x d t \\
& =\int_{0}^{\infty} \int_{0}^{\infty} e^{-\left(\frac{(p-a u) x}{u}+\frac{(q-b v) t}{v}\right)} d x d t \\
& =\frac{u v}{(p-a u)(q-b v)}
\end{aligned}
$$


(3) If $g(x, t)=e^{i(a x+b t)}$, then

$$
\begin{aligned}
H_{x t}^{2}(g(x, t)) & -\frac{u v}{(p-a u i)(q-b v i)} \\
& =\frac{u v(p q+a b u v)+(b p v+a q u) u v i}{\left(p^{2}+a^{2} u^{2}\right)\left(q^{2}+b^{2} v^{2}\right)} .
\end{aligned}
$$

As a consequence of property (3)

$$
\begin{aligned}
& H_{x t}^{2}(\cos (a x+b t))=\frac{u v(p q+a b u v)}{\left(p^{2}+a^{2} u^{2}\right)} \\
& H_{x t}^{2}(\sin (a x+b t))=\frac{u v(b p v+a q u)}{\left(q^{2}+b^{2} v^{2}\right)} .
\end{aligned}
$$

(4) If $g(x, t)=\cosh (a x+b t)$, then

$$
\begin{aligned}
H_{x t}^{2}(g(x, t)) & =\frac{1}{2}\left[H_{x t}^{2}\left(e^{(a x+b t)}\right)+H_{x t}^{2}\left(e^{-(a x+b t)}\right)\right] \\
& =\frac{1}{2}\left[\frac{u v}{(p-a u)(q-b v)}+\frac{u v}{(p+a u)(q+b v)}\right] .
\end{aligned}
$$

Similarly,

$$
H_{x t}^{2}(\sinh (a x+b t))=\frac{1}{2}\left[\frac{u v}{(p-a u)(q-b v)}-\frac{u v}{(p+a u)(q+b v)}\right] .
$$

(5) If $g(x, t)=x^{n} t^{m}, n, m=0,1,2, \cdots$, then

$$
\begin{aligned}
H_{x t}^{2}(g(x, t)) & =\int_{0}^{\infty} \int_{0}^{\infty} e^{-\left(\frac{p x}{u}+\frac{q t}{v}\right)} x^{n} t^{m} d x d t \\
& =\int_{0}^{\infty} e^{-\left(\frac{p x}{u}\right)} x^{n} d x \int_{0}^{\infty} e^{-\left(\frac{q t}{v}\right)} t^{m} d x d t \\
& =n ! m !\left(\frac{u}{p}\right)^{n+1}\left(\frac{v}{q}\right)^{m+1}
\end{aligned}
$$

(6) If $g(x, t)=x^{\alpha} t^{\gamma}, \alpha \geq-1, \gamma \geq-1$ then

$$
\begin{aligned}
H_{x t}^{2}(g(x, t)) & =\int_{0}^{\infty} \int_{0}^{\infty} e^{-\left(\frac{p x}{u}+\frac{q t}{v}\right)} x^{\alpha} t^{\gamma} d x d t \\
& =\int_{0}^{\infty} e^{-\left(\frac{p x}{u}\right)} x^{\alpha} d x \int_{0}^{\infty} e^{-\left(\frac{q t}{v}\right)} t^{\gamma} d t
\end{aligned}
$$

by letting $\mathrm{y}=\left(\frac{p x}{u}\right)$, and $z=\left(\frac{q t}{v}\right)$

$$
\begin{aligned}
& =\left(\frac{u}{p}\right)^{\alpha+1} \int_{0}^{\infty} e^{-y} y^{\alpha} d y\left(\frac{v}{q}\right)^{\gamma+1} \int_{0}^{\infty} e^{-z} z^{\gamma} d z \\
& =\Gamma(\alpha+1)\left(\frac{u}{p}\right)^{\alpha+1} \Gamma(\gamma+1)\left(\frac{v}{q}\right)^{\gamma+1} .
\end{aligned}
$$

Where, $\Gamma($.$) is the Euler gamma function.$ 


\section{Properties of the Double Shehu Transform}

(1) The double Shehu transform $H_{x t}^{2}($.$) is a linear operator, that is$

$$
H_{x t}^{2}[(a g+b h)(x, t)]=a H_{x t}^{2}[g(x, t)]+b H_{x t}^{2}[h(x, t)]
$$

\section{Proof.}

$$
\begin{aligned}
H_{x t}^{2}[(a g+b h)(x, t)] & =\int_{0}^{\infty} \int_{0}^{\infty} e^{-\left(\frac{p x}{u}+\frac{q t}{v}\right)}(a g+b h) d x d t \\
& =a \int_{0}^{\infty} \int_{0}^{\infty} e^{-\left(\frac{p x}{u}+\frac{q t}{v}\right)} g(x, t) d x d t+b \int_{0}^{\infty} \int_{0}^{\infty} e^{-\left(\frac{p x}{u}+\frac{q t}{v}\right)} h(x, t) d x d t \\
& =a H_{x t}^{2}[g(x, t)]+b H_{x t}^{2}[h(x, t)]
\end{aligned}
$$

(2) Changing of scale property

If $H_{x t}^{2}(g(x, t))=G((p, q),(u, v))$, then $H_{x t}^{2}(g(a x, b t))=\frac{1}{a b} G\left(\left(\frac{p}{a}, \frac{q}{b}\right),(u, v)\right)$.

Proof. Using the definition of (DHT), we deduce

$$
H_{x t}^{2}(g(a x, b t))=\int_{0}^{\infty} \int_{0}^{\infty} e^{-\left(\frac{p x}{u}+\frac{q t}{v}\right)} g(a x, b t) d x d t
$$

Substituting $y=a x$, and $z=b t$ in equation (4.1), we get

$$
\begin{aligned}
& =\frac{1}{a b} \int_{0}^{\infty} \int_{0}^{\infty} e^{-\left(\frac{p y}{u a}+\frac{q z}{v b}\right)} g(y, z) d y d z \\
& =\frac{1}{a b} \int_{0}^{\infty} \int_{0}^{\infty} e^{-\left(\frac{\left(\frac{p}{a}\right) y}{u}+\frac{\left(\frac{q}{b}\right) z}{v}\right)} g(y, z) d y d z \\
& =\frac{1}{a b} G\left(\left(\frac{p}{a}, \frac{q}{b}\right),(u, v)\right) .
\end{aligned}
$$

(3) Shifting property

If $H_{x t}^{2}(g(x, t))=G((p, q),(u, v))$, then $H_{x t}^{2}\left(e^{-(a x+b t)} g(x, t)\right)=G((p+a u, q+b v),(u, v))$.

Proof. Using the definition of (DHT), we deduce

$$
\begin{aligned}
H_{x t}^{2}\left(e^{-(a x+b t)} g(x, t)\right) & =\int_{0}^{\infty} \int_{0}^{\infty} e^{-\left(\frac{p x}{u}+\frac{q t}{v}\right)} e^{-(a x+b t)} g(x, t) d x d t \\
& =\int_{0}^{\infty} \int_{0}^{\infty} e^{-\left(\frac{(p+a u) x}{u}+\frac{(q+b v) t}{v}\right)} g(x, t) d x d t \\
& =G((p+a u, q+b v),(u, v))
\end{aligned}
$$

Theorem 4.1. If $H_{x t}^{2}(g(x, t))=G((p, q),(u, v))$, Then

$$
H_{x t}^{2}[g(x-a, t-b) H(x-a, t-b)]=e^{-a \frac{p}{u}-b \frac{q}{v}} G((p, q),(u, v)),
$$


where $H(x, t)$ is the Heaviside unit step function defined as follows :

$$
H(x-a, t-b)=\left\{\begin{array}{cc}
1 & x>a, t>b \\
0 & \text { otherwise }
\end{array}\right.
$$

Proof: By using the definition of (DHT), we have

$$
\begin{aligned}
H_{x t}^{2}[g(x-a, t-b) H(x-a, t-b)] & =\int_{0}^{\infty} \int_{0}^{\infty} e^{-\left(\frac{p x}{u}+\frac{q t}{v}\right)} g(x-a, t-b) H(x-a, t-b) d x d t \\
& =\int_{a}^{\infty} \int_{b}^{\infty} e^{-\left(\frac{p x}{u}+\frac{q t}{v}\right)} g(x-a, t-b) d x d t
\end{aligned}
$$

By substituting $x-a=y, \quad t-b=z$, we get

$$
\begin{aligned}
& =\int_{a}^{\infty} \int_{b}^{\infty} e^{-\left(\frac{p(y+a)}{u}+\frac{q(z+b)}{v}\right)} g(y, z) d y d z \\
& =e^{-\left(\frac{a p}{u}+\frac{b q}{v}\right)} \int_{a}^{\infty} \int_{b}^{\infty} e^{-\left(\frac{p y}{u}+\frac{q z}{v}\right)} g(y, z) d y d z \\
& =e^{-\left(\frac{a p}{u}+\frac{b q}{v}\right)} H_{x t}^{2}(g(x, t))
\end{aligned}
$$

(4) The double Shehu transform of the first and the second order partial derivatives with respect to $x$

$$
\begin{aligned}
& H_{x t}^{2}\left(\frac{\partial g}{\partial x}\right)=\left(\frac{p}{u}\right) G^{2}((p, q),(u, v))-H_{t}(g(0, t)), \\
& H_{x t}^{2}\left(\frac{\partial^{2} g}{\partial x^{2}}\right)=\left(\frac{p}{u}\right)^{2} G^{2}((p, q),(u, v))-\left(\frac{p}{u}\right) H_{t}(g(0, t))-H_{t}\left(\frac{\partial}{\partial x} g(0, t)\right) .
\end{aligned}
$$

Similarly, with respect to $t$

$$
\begin{aligned}
& H_{x t}^{2}\left(\frac{\partial g}{\partial t}\right)=\left(\frac{q}{v}\right) G((p, q),(u, v))-H_{x}(g(x, 0)), \\
& H_{x t}^{2}\left(\frac{\partial^{2} g}{\partial t^{2}}\right)=\left(\frac{q}{v}\right)^{2} G((p, q),(u, v))-\left(\frac{q}{v}\right) H_{x}(g(x, 0))-H_{x}\left(\frac{\partial}{\partial t} g(x, 0)\right) .
\end{aligned}
$$

Moreover, the double Shehu transform for the mixed double order partial derivative of function of two variables is given by

$$
H_{x t}^{2}\left(\frac{\partial^{2} g}{\partial x \partial t}\right)=\left(\frac{p q}{u v}\right) G((p, q),(u, v))-\left(\frac{q}{v}\right) H_{t}(g(0, t))-\left(\frac{p}{u}\right) H_{x}(g(x, 0))+g(0,0) .
$$

Theorem 4.2. The double Shehu transforms of the, $n, m \in \mathrm{N}$ times partial derivatives $\frac{\partial^{n} g}{\partial x^{n}}, \frac{\partial^{m} g}{\partial t^{m}}$, of the function $g(x, t)$ are given by:

$$
\begin{aligned}
& H_{x t}^{2}\left(\frac{\partial^{n} g}{\partial x^{n}}\right)=\left(\frac{p}{u}\right)^{n} G((p, q),(u, v))-\left(\frac{p}{u}\right)^{n-1} H_{t}(g(0, t))-\sum_{j=1}^{n-1}\left(\frac{p}{u}\right)^{n-1-j} H_{t}\left(\frac{\partial^{j}}{\partial x^{j}} g(0, t)\right), \\
& H_{x t}^{2}\left(\frac{\partial^{m} g}{\partial t^{m}}\right)=\left(\frac{q}{v}\right)^{n} G((p, q),(u, v))-\left(\frac{q}{v}\right)^{m-1} H_{x}(g(x, 0))-\sum_{j=1}^{m-1}\left(\frac{q}{v}\right)^{m-1-j} H_{x}\left(\frac{\partial^{j}}{\partial t^{j}} g(x, 0)\right) .
\end{aligned}
$$

Proof. the proof can be done by mathematical induction. 
Theorem 4.3. If the double Shehu transform of $\frac{\partial^{n} g}{\partial x^{n}}, \frac{\partial^{m} g}{\partial t^{m}}$ is given by equations (4.2) then the double Shehu transforms of

$$
x^{m} \frac{\partial^{n} g(x, t)}{\partial x^{n}}, \text { and } t^{m} \frac{\partial^{m} g(x, t)}{\partial t^{m}}
$$

are given by

$$
\begin{gathered}
H_{x t}^{2}\left(x^{n} \frac{\partial^{n} g(x, t)}{\partial x^{n}}\right)=(-1)^{n} u^{n} \frac{\partial^{n}}{\partial p^{n}}\left(H_{x t}^{2}(g(x, t))\right) \\
H_{x t}^{2}\left(t^{m} \frac{\partial^{m} g(x, t)}{\partial t^{m}}\right)=(-1)^{m} v^{m} \frac{\partial^{m}}{\partial q^{m}}\left(H_{x t}^{2}(g(x, t))\right), \text { where } m, n=1,2,3, \ldots
\end{gathered}
$$

Proof. By using the definition of (DST), we get

$$
H_{x t}^{2}(g(x, t))=\int_{0}^{\infty} \int_{0}^{\infty} e^{-\left(\frac{p x}{u}+\frac{q t}{v}\right)} g(x, t) d x d t,
$$

taking the $n^{\text {th }}$ partial derivative w.r.t $p$ for both sides of equation (4.5), we have

$$
\begin{aligned}
\frac{\partial^{n}}{\partial p^{n}}\left(H_{x t}^{2}(g(x, t))\right) & =\int_{0}^{\infty} \int_{0}^{\infty} \frac{\partial^{n}}{\partial p^{n}}\left[e^{-\left(\frac{p x}{u}+\frac{q t}{v}\right)}\right] g(x, t) d x d \\
& =(-1)^{n} \int_{0}^{\infty} \int_{0}^{\infty}\left(\frac{x}{u}\right)^{n} e^{-\left(\frac{p x}{u}+\frac{q t}{v}\right)} g(x, t) d x d \\
& =(-1)^{n} \frac{1}{u^{n}} \int_{0}^{\infty} \int_{0}^{\infty} x^{n} e^{-\left(\frac{p x}{u}+\frac{q t}{v}\right)} g(x, t) d x d \\
& =(-1)^{n} \frac{1}{u^{n}}\left(H_{x t}^{2}\left(x^{n} g(x, t)\right)\right)
\end{aligned}
$$

simplifying, we obtain

$$
(-1)^{n} u^{n} \frac{\partial^{n}}{\partial p^{n}}\left(H_{x t}^{2}(g(x, t))\right)=H_{x t}^{2}\left(x^{n} g(x, t)\right) .
$$

Similarly, equation (4.4) can be proven.

Theorem 4.4. (Double Shehu-Double Laplace duality). If the (DHT) of a function $g(x, t)$ exists, then

$$
H_{x t}^{2}(g(x, t)(p, q),(u, v))=L_{x t}^{2}\left(g(x, t),\left(\frac{p}{u}, \frac{q}{v}\right)\right),
$$

where $L_{x t}^{2}$ denotes the $(D L T)$ of the function $g(x, t)$.

Proof. The proof can be done directly from the definition of (DHT) that is

$$
H_{x t}^{2}(g(x, t)(p, q),(u, v))=\int_{0}^{\infty} \int_{0}^{\infty} e^{-\left(\frac{p x}{u}+\frac{q t}{v}\right)} g(x, t) d x d t=L_{x t}^{2}\left(g(x, t),\left(\frac{p}{u}, \frac{q}{v}\right)\right) .
$$


Theorem 4.5. (Double Shehu-Double Sumudu duality). If the (DHT) of a function $g(x, t)$ exists, then

$$
H_{x t}^{2}(g(x, t)(p, q),(u, v))=\frac{u v}{p q} S_{x t}^{2}\left(g(x, t),\left(\frac{u}{p}, \frac{v}{q}\right)\right),
$$

where $S_{x t}^{2}$ denotes the (DST) of the function $g(x, t)$.

Proof. By letting $\phi=\frac{p x}{u}, \varphi=\frac{q t}{v}$ in the (DHT) formula, we get

$$
H_{x t}^{2}(g(x, t)(p, q),(u, v))=\frac{u v}{p q} \int_{0}^{\infty} \int_{0}^{\infty} e^{-(y+y)} g\left(\frac{u y}{p}, \frac{v z}{q}\right) d y d z=\frac{u v}{p q} S_{x t}^{2}\left(g(x, t),\left(\frac{u}{p}, \frac{v}{q}\right)\right) .
$$

Theorem 4.6. Let $g(x, t)$, and $h(x, t)$ be of exponential order, having double Shehu transforms $H_{x t}^{2}(g(x, t))$, and $H_{x t}^{2}(h(x, t))$, respectively. The the double Shehu transform of the convolution of $g(x, t)$ and $h(x, t)$

$$
[g * * h](x, t)=\int_{0}^{x} \int_{0}^{t} g(x-\kappa, t-\lambda) h(\kappa, \lambda) d \kappa d \lambda,
$$

is given by

$$
H_{x t}^{2}([g * * h](x, t))=H_{x t}^{2}(g(x, t)) H_{x t}^{2}(h(x, t))
$$

Proof. Using theorem (4.5), we have

$$
\begin{aligned}
H_{x t}^{2}([g * * h](x, t)) & =\frac{u v}{p q} S_{x t}^{2}([g * * h](x, t)) \\
& =\left(\frac{u v}{p q}\right)^{2} S_{x t}^{2}(g(x, t)) S_{x t}^{2}(h(x, t)) \\
& =\left(\frac{u v}{p q}\right) S_{x t}^{2}(g(x, t))\left(\frac{u v}{p q}\right) S_{x t}^{2}(h(x, t)) \\
& =H_{x t}^{2}(g(x, t)) H_{x t}^{2}(h(x, t)) .
\end{aligned}
$$

\section{Application of (DHT) method to integral and partial differential EQuations}

In this section, we illustrate the applicability of the (DHT) method, by constructing some examples.

Example 5.1. Consider the following equation of Volterra Integro PDE.

$$
\frac{\partial}{\partial x} g(x, t)+\frac{\partial}{\partial t} g(x, t)+1-e^{x}-e^{t}-e^{x+t}=\int_{0}^{x} \int_{0}^{t} g(\kappa, \lambda) d \kappa d \lambda,
$$

with respect to the intial conditions

$$
g(x, 0)=e^{x}, \quad g(0, t)=e^{t}
$$

Applying (DHT) for equation (5.1), we get,

$$
\begin{aligned}
& \left(\frac{p}{u}\right) G^{2}((p, q),(u, v))-H_{t}(g(0, t))+\left(\frac{q}{v}\right) G^{2}((p, q),(u, v))-H_{x}(g(x, 0)) \\
& +\frac{u v}{p q}-\frac{u v}{(p-u) q}-\frac{u v}{(q-v) p}-\frac{u v}{(p-u)(q-v)}=H_{x t}^{2}([g * * 1](x, t)) .
\end{aligned}
$$


The single Shehu transform of the initial conditions

$$
H_{x}(g(x, 0))=\frac{u}{(p-u)}, H_{t}(g(0, t))=\frac{v}{(q-v)} .
$$

Substituting equation (5.4) into equation(5.3), we get

$$
\begin{aligned}
& \left(\frac{p}{u}\right) G^{2}((p, q),(u, v))-\frac{u}{(p-u)}+\left(\frac{q}{v}\right) G^{2}((p, q),(u, v))-\frac{v}{(q-v)} \\
& +\frac{u v}{p q}-\frac{u v}{(p-u) q}-\frac{u v}{(q-v) p}-\frac{u v}{(p-u)(q-v)}=\left(\frac{u v}{p q}\right) G^{2}((p, q),(u, v)) .
\end{aligned}
$$

Simplifying, we get

$$
G^{2}((p, q),(u, v))=\frac{u v}{(p-u)(q-v)},
$$

taking the inverse double Shehu transform of equation (5.6)

$$
g(x, t)=H_{x t}^{-2}\left[\frac{u v}{(p-u)(q-v)}\right]=e^{x+t} .
$$

Example 5.2. Consider the following partial double integro-differential equation.

$$
\frac{\partial^{2}}{\partial t^{2}} g(x, t)-\frac{\partial^{2}}{\partial x^{2}} g(x, t)+g(x, t)+\int_{0}^{x} \int_{0}^{t} e^{x-\kappa+t-\lambda} g(\kappa, \lambda) d \kappa d \lambda=x t e^{x+t}+e^{x+t},
$$

with respect to the initial conditions

$$
g(x, 0)=e^{x}, \quad \frac{\partial}{\partial x} g(x, 0)=e^{x}, \quad g(0, t)=e^{t}, \quad \frac{\partial}{\partial t} g(0, t)=e^{t} .
$$

Taking the (DHT) to both side of equation (5.8), we get

$$
\begin{aligned}
& \left(\frac{q}{v}\right)^{2} G^{2}((p, q),(u, v))-\left(\frac{q}{v}\right) H_{x}(g(x, 0))-H_{x}\left(\frac{\partial}{\partial t} g(x, 0)\right)-\left(\frac{p}{u}\right)^{2} G^{2}((p, q),(u, v)) \\
& -\left(\frac{p}{u}\right) H_{t}(g(0, t))-H_{t}\left(\frac{\partial}{\partial x} g(0, t)\right)+G^{2}((p, q),(u, v))+H_{x t}^{2}\left(\left[e^{x+t} * * g\right](x, t)\right) \\
& =\frac{u^{2} v^{2}}{(p-u)^{2}(q-v)^{2}}+\frac{u v}{(p-u)(q-v)},
\end{aligned}
$$

substituting the single Shehu transform of initial and boundary conditions,

$$
H_{x}(g(x, 0))=\frac{u}{(p-u)}, H_{x}\left(\frac{\partial}{\partial x} g(x, 0)\right)=\frac{u}{(p-u)}, H_{t}(g(0, t))=\frac{v}{(q-v)}, H_{t}\left(\frac{\partial}{\partial t} g(0, t)\right)=\frac{v}{(q-v)},
$$

in equation (5.10) we get

$$
\begin{aligned}
& {\left[\left(\frac{q}{v}\right)^{2}-\left(\frac{p}{u}\right)^{2}+1+\frac{u v}{(p-u)(q-v)}\right] G^{2}((p, q),(u, v))=\frac{q u}{(p-u) v}+\frac{u}{(p-u)}+\frac{p v}{(q-v) u}+\frac{v}{(q-v)}} \\
& +\frac{u^{2} v^{2}}{(p-u)^{2}(q-v)^{2}}+\frac{u v}{(p-u)(q-v)}
\end{aligned}
$$


simplifying, we get

$$
G^{2}((p, q),(u, v))=\frac{u v}{(p-u)(q-v)},
$$

taking the inverse double Shehu transform of equation (5.12)

$$
g(x, t)=H_{x t}^{-2}\left[\frac{u v}{(p-u)(q-v)}\right]=e^{x+t} .
$$

Example 5.3. Consider the following partial integro-differential equation.

$$
\frac{\partial^{2}}{\partial x \partial t} g(x, t)+g(x, t)=\frac{-1}{4} x^{2} t^{2}+x t+1+\int_{0}^{x} \int_{0}^{t} g(\kappa, \lambda) d \kappa d \lambda,
$$

with respect to the initial conditions

$$
g(x, 0)=0, \quad g(0, t)=0 .
$$

Taking the (DHT) to both side of equation (5.15), we get

$$
\begin{aligned}
& \left(\frac{p q}{u v}\right) G((p, q),(u, v))-\left(\frac{q}{v}\right) H_{t}(g(0, t))-\left(\frac{p}{u}\right) H_{x}(g(x, 0))+g(0,0)+ \\
& G^{2}((p, q),(u, v))=-\frac{u^{3} v^{3}}{p^{3} q^{3}}+\frac{u^{2} v^{2}}{p^{2} q^{2}}+\frac{u v}{p q}+H_{x t}^{2}([1 * * g](x, t)),
\end{aligned}
$$

substituting the single Shehu transform of initial and boundary conditions,

$$
H_{t}(g(0, t))=0, H_{x}(g(x, 0))=0,
$$

in equation (5.16) we get

$$
\left[\frac{p q}{u v}+1-\frac{u v}{p q}\right] G^{2}((p, q),(u, v))=-\frac{u^{3} v^{3}}{p^{3} q^{3}}+\frac{u^{2} v^{2}}{p^{2} q^{2}}+\frac{u v}{p q},
$$

simplifying, we get

$$
G^{2}((p, q),(u, v))=\frac{u^{2} v^{2}}{p^{2} q^{2}}
$$

taking the inverse double Shehu transform of equation (5.18)

$$
g(x, t)=H_{x t}^{-2}\left[\frac{u^{2} v^{2}}{p^{2} q^{2}}\right]=x t .
$$

Example 5.4. Consider the following Partial differential Telegraph equation

$$
\frac{\partial^{2} g(x, t)}{\partial x^{2}}-\frac{\partial^{2} g(x, t)}{\partial t^{2}}-\frac{\partial g(x, t)}{\partial t}-g+x^{2}+t=1,
$$

with respect to the initial and boundary conditions

$$
\begin{aligned}
& g(x, 0)=x^{2}, \quad \frac{\partial}{\partial t} g(x, 0)=1, \\
& g(0, t)=t, \quad \frac{\partial}{\partial x} g(0, t)=0 .
\end{aligned}
$$


Applying the (DHT) to both side of equation (5.20) and single (HT) to the initial and boundary conditions (5.21), we get

$$
\begin{aligned}
& \left(\frac{p}{u}\right)^{2} G^{2}((p, q),(u, v))-\left(\frac{p}{u}\right) H_{t}(g(0, t))-H_{t}\left(\frac{\partial}{\partial x} g(0, t)\right)-\left(\frac{q}{v}\right)^{2} G^{2}((p, q),(u, v))+\left(\frac{q}{v}\right) H_{x}(g(x, 0)) \\
& +H_{x}\left(\frac{\partial}{\partial t} g(x, 0)\right)-\left(\frac{q}{v}\right) G^{2}((p, q),(u, v))+H_{x}(g(x, 0))-G^{2}((p, q),(u, v))+\frac{u^{3} v}{p^{3} q}+\frac{u v^{2}}{p q^{2}}=\frac{u}{p} .
\end{aligned}
$$

Substituting the single (HT) of initial and boundary conditions

$$
H_{x}(g(x, 0))=\frac{u^{3}}{p^{3}}, H_{x}\left(\frac{\partial}{\partial t} g(x, 0)\right)=\frac{u}{p}, H_{t}(g(0, t))=\frac{v^{2}}{q^{2}}, H_{t}\left(\frac{\partial}{\partial x} g(0, t)\right)=0,
$$

in equation (5.22), we get

$$
\left[\left(\frac{p}{u}\right)^{2}-\left(\frac{q}{v}\right)^{2}-\left(\frac{q}{v}\right)-1\right] G^{2}((p, q),(u, v))=\frac{p v^{2}}{u q^{2}}-\frac{q u^{3}}{v p^{3}}-\frac{u}{p}-\frac{u^{3}}{p^{3}}-\frac{u^{3} v}{p^{3} q}-\frac{u v^{2}}{p q^{2}}+\frac{u}{p},
$$

simplifying, we obtain

$$
G^{2}((p, q),(u, v))=\frac{u v^{2}}{p q^{2}}+2 \frac{u^{3}}{p^{3}} \frac{v^{3}}{q^{3}}
$$

taking the inverse of (DHT), we get

$$
g(x, t)=H_{x t}^{-2}\left[\frac{u v^{2}}{p q^{2}}+2 \frac{u^{3}}{p^{3}} \frac{v^{3}}{q^{3}}\right]=t+x^{2} .
$$

Example 5.5. Consider the Korteweg- de Vries PDE.

$$
\frac{\partial^{3} g}{\partial x^{3}}+\frac{\partial g}{\partial x}+\frac{\partial g}{\partial t}=0
$$

with respect to the initial and boundary conditions

$$
g(x, 0)=e^{-x}, \quad g(0, t)=e^{2 t}, \quad \frac{\partial}{\partial x} g(0, t)=-e^{2 t}, \quad \frac{\partial^{2}}{\partial x^{2}} g(0, t)=e^{2 t} .
$$

Applying the (DHT) to both side of equation (5.24) and single (HT) to initial and boundary conditions (5.25), we get

$$
\begin{aligned}
& \left(\frac{p}{u}\right)^{3} G^{2}((p, q),(u, v))-\left(\frac{p}{u}\right)^{2} H_{t}(g(0, t))-\left(\frac{p}{u}\right) H_{t}\left(\frac{\partial}{\partial x} g(0, t)\right)-H_{t}\left(\frac{\partial^{2}}{\partial x^{2}} g(0, t)\right) \\
& +\left(\frac{p}{u}\right) G^{2}((p, q),(u, v))-H_{t}(g(0, t))+\left(\frac{q}{v}\right) G^{2}((p, q),(u, v))-H_{x}(g(x, 0))=0,
\end{aligned}
$$

substituting the single (HT) of the initial and boundary conditions

$$
H_{x}(g(x, 0))=\frac{u}{p+u}, \quad H_{t}(g(0, t))=\frac{v}{q-2 v}, H_{t}\left(\frac{\partial}{\partial x} g(0, t)\right)=\frac{-v}{q-2 v}, H_{t}\left(\frac{\partial^{2}}{\partial x^{2}} g(0, t)\right)=\frac{v}{q-2 v},
$$

in equation (5.26), we obtain

$$
\left[\left(\frac{p}{u}\right)^{3}+\left(\frac{p}{u}\right)+\left(\frac{q}{v}\right)\right] G^{2}((p, q),(u, v))=\left(\frac{p}{u}\right)^{2} \frac{v}{q-2 v}-\left(\frac{p}{u}\right) \frac{v}{q-2 v}+\frac{v}{q-2 v}+\frac{v}{q-2 v}+\frac{u}{p+u},
$$

simplifying,

$$
G^{2}((p, q),(u, v))=\left(\frac{u}{p+u} \frac{v}{q-2 v}\right)
$$


taking the inverse of (DHT), we get

$$
g(x, t)=H_{x t}^{-2}\left[\frac{u}{p+u} \frac{v}{q-2 v}\right]=e^{-x+2 t} .
$$

Example 5.6. Consider the partial differential Euler -Bernoulli equation

$$
\frac{\partial^{4} g}{\partial x^{4}}+\frac{\partial^{2} g}{\partial t^{2}}=t^{2}+x t
$$

with respect to the initial and boundary conditions

$$
\begin{aligned}
& g(x, 0)=0, \quad \frac{\partial}{\partial t} g(x, 0)=\frac{1}{120} x^{5}, \\
& g(0, t)=\frac{1}{12} t^{4}, \quad \frac{\partial^{i}}{\partial x^{i}} g(0, t)=0, \quad \text { for }, i=1,2,3 .
\end{aligned}
$$

Applying the (DHT) to both side of equation (5.28) and single (HT) to initial and boundary conditions (5.29), we get

$$
\begin{aligned}
& \left(\frac{p}{u}\right)^{4} G^{2}((p, q),(u, v))-\left(\frac{p}{u}\right)^{3} H_{t}(g(0, t))-\left(\frac{p}{u}\right)^{2} H_{t}\left(\frac{\partial}{\partial x} g(0, t)\right) H_{t}(g(0, t))-\left(\frac{p}{u}\right) H_{t}\left(\frac{\partial^{2}}{\partial x^{2}} g(0, t)\right) \\
& -H_{t}\left(\frac{\partial^{3}}{\partial x^{3}} g(0, t)\right)+\left(\frac{q}{v}\right)^{2} G^{2}((p, q),(u, v))-\left(\frac{q}{v}\right) H_{x}(g(x, 0))-H_{x}\left(\frac{\partial}{\partial t} g(x, 0)\right)=\frac{2 u v^{2}}{p q^{2}}+\frac{u v}{p q},
\end{aligned}
$$

substituting,

$$
H_{x}(g(x, 0))=0, \quad H_{t}\left(\frac{\partial}{\partial t} g(x, 0)\right)=\left(\frac{u}{p}\right)^{6}, H_{t}(g(0, t))=\left(\frac{v}{q}\right)^{5}, H_{t}\left(\frac{\partial^{i}}{\partial x^{i}} g(0, t)\right)=0, i=1,2,3,
$$

in equation (5.30), we obtain

$$
\left[\left(\frac{p}{u}\right)^{4}+\left(\frac{q}{v}\right)^{2}\right] G^{2}((p, q),(u, v))=\left(\frac{p}{u}\right)^{3}\left(\frac{v}{q}\right)^{5}+\left(\frac{u}{p}\right)^{6}+\frac{2 u v^{2}}{p q^{2}}+\frac{u v}{p q},
$$

simplifying,

$$
G^{2}((p, q),(u, v))=\left(\frac{2 u v^{4}}{p q^{4}}+\frac{v^{2} u^{6}}{q^{2} p^{6}}\right) .
$$

Taking the inverse of (DHT), we get

$$
g(x, t)=H_{x t}^{-2}\left[\frac{2 u v^{4}}{p q^{4}}+\frac{v^{2} u^{6}}{q^{2} p^{6}}\right]=\frac{2 t^{4}}{4 !}+\frac{t x^{5}}{5 !} .
$$

\section{Conclusions}

In this study, we have extended the work of [11], to the double Shehu transform (DHT). First, we have discussed and proved the existence and uniqueness of the double Shehu transform. Next, Some Fundamental properties and theorems using the new double Shehu transform have also been presented. It is analyzed that the devolved method is well suited for use in integral and partial differential equations involving two variables. Therefore, the proposed method is an efficient, accurate and reliable technique for the integrals and partial differential equations. Finally, it is worthwhile to mention that the (DHT) can be coupled with some 
other methods to solve non-linear (PDEs) arising in applied mathematics, applied physics and engineering, which will be discussed in subsequent articles.

Conflicts of Interest: The author(s) declare that there are no conflicts of interest regarding the publication of this paper.

\section{REFERENCES}

[1] R. N. Bracewell, The Fourier Transform and Its Applications (3rd ed.), McGraw-Hill, New York, (1986).

[2] D. V. Widder, The Laplace transform, Princeton University Press, Princeton, (1946).

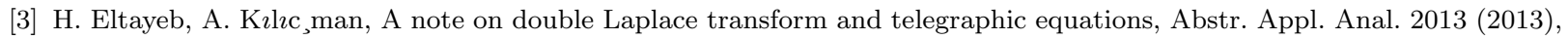
Article ID 932578.

[4] G. K. Watugala, Sumudu transform: a new integral transform to solve differential equations and control engineering problems, Int. J. Math. Educ. Sci. Technol. 24 (1993), 35-43.

[5] F. B. M. Belgacem, A. A. Karaballi, Sumudu transform fundamental properties investigations and applications, J. Appl. Math. Stoch. Anal. 2006 (2006), Article ID 91083.

[6] M. A. Asiru, Sumudu transform and the solution of integral equations of convolution type, Int. J. Math. Educ. Sci. Technol. $32(2001), 906-910$.

[7] S. K. Q. Al-Omari, On the application of natural transforms, Int. J. Pure Appl. Math. 85 (2013), 729-744.

[8] T. M. Elzaki, The new integral transform "Elzaki transform", Glob. J. Pure Appl. Math. 7(1) (2011), 57-64.

[9] P. K. G. Bhadane, V.H. Pradhan and S. V. Desale, Elzaki transform solution of one dimensional groundwater recharge through spreading, Int. J. Eng. Res. Appl.3 (6) (2013), 1607-1610.

[10] L. Debnath and D. Bhatta, Integral Transforms and Their Applications, CRC Press, Taylor Francis Group, Boca Raton, Fla, USA, 3rd edition, (2015).

[11] S. Maitama and W. Zhao, New Integral Transform: Shehu Transform a Generalization of Sumudu and Laplace Transform for Solving Differential Equations, Int. J. Anal. Appl. 17 (2) (2019), 167-190.

[12] A. Khalouta and A. Kade, A New Method to Solve Fractional Differential Equations: Inverse Fractional Shehu Transform Method, Appl. Appl. Math. 14 (2) (2019), 926-941.

[13] Aggarwal, Gupta, A.R and S., Sharma, N. A New Application of Shehu Transform for Handling Volterra Integral Equations of First Kind, Int. J. Res. Advent Technol. 7 (4) (2019), 438-445.

[14] A. Bokhari, D. Baleanu, and R. Belgacem. Application of Shehu transform to Atangana-Baleanu derivatives. J. Math. Computer Sci. 20 (2019), 101-107.

[15] R. Belgacem, D. Baleanu, and A. Bokhari, Shehu Transform and Applications to Caputo-Fractional Differential Equations. Int. J. Anal. Appl. 17 (6) (2019), 917-927.

[16] Dhunde, Ranjit R. and Waghmare, G.L. . Solving partial integro-differential equations using double Laplace transform method, Amer. J. Comput. Appl. Math. 5 (1) (2015), 7-10.

[17] Eltayeb, Hassan and Kilicman, Adem, On double Sumudu transform and double Laplace transform, Malaysian J. Math. Sci. 4 (1) (2010), 17-30.

[18] H. Eltayeb, A. Kılıçman, A note on the Sumudu transforms and differential equations, Appl. Math. Sci. 4 (22) (2010), 1089-1098.

[19] G. K. Watugala, The Sumudu transform for functions of two variables, Math. Eng. Ind. 8 (2002), 293-302. 
[20] A. Kılıçman, H. Eltayeb, A note on integral transforms and partial differential equations, Appl. Math. Sci. 4 (2010), 109-118.

[21] Wazwaz, Abdul Majid . Partial Differential Equations and Solitary Waves Theory, Higher Education Press Beijing and Springer-Verlag, Berlin Heidelberg (2009).

[22] G. L. Lamb Jr, Introductory Applications of Partial Differential Equations with Emphasis on Wave Propagation and Diffusion, John Wiley \& Sons, New York, NY, USA, (1995). 